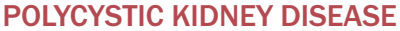

\section{Role of TRPV4 in renal cystogenesis}

The increase in intracellular $\mathrm{Ca}^{2+}$ levels that occurs in collecting duct (CD) cells in response to alterations in tubular flow may be mediated by transient receptor potential cation channel subfamily V member 4 (TRPV4). Now, Oleh Pochynyuk and colleagues report that disrupted functioning of this mechanosensitive channel in CD cells might have a role in cyst formation in autosomal recessive polycystic kidney disease (ARPKD).

To investigate TRPV4 function and mechanosensitive $\mathrm{Ca}^{2+}$ signalling in renal cysts, the researchers isolated cyst cell monolayers and nondilated cells from the CDs of rats with ARPKD. "Not all renal tubules transform into cysts during polycystic kidney disease and the mechanisms triggering this transformation are poorly understood," says Pochynyuk. "Using our experimental approach we were able to isolate cyst monolayers and nondilated CDs from the same kidney and compare their functional properties."
The researchers found reduced levels of basal $\mathrm{Ca}^{2+}$, abnormal subcellular localization of TRPV4, and impaired TRPV4 activation and flow-mediated $\mathrm{Ca}^{2+}$ signalling in CD cyst cells from ARPKD rats compared with epithelial cells from nondilated CDs from ARPKD rats and control Sprague-Dawley rats. Long-term pharmacological activation of TRPV4 restored mechanosensitive $\mathrm{Ca}^{2+}$ signalling and reduced the development and growth of renal cysts in ARPKD rats.

The researchers conclude that disruption of TRPV4 activity might occur before, or be an early event in cyst formation. They suggest that TRPV4 activity could be a therapeutic target for the prevention of ARPKD progression.

Ellen F. Carney

Original article Zaika, 0. et al. TRPV4 dysfunction promotes renal cystogenesis in autosomal recessive polycystic kidney disease. J.Am. Soc. Nephrol. doi:10.1681/ ASN.2012050442 